\title{
Effects of claudin-1 downregulation on the physiological processes of gallbladder cancer SGC996 cells
}

\author{
HAO JIN $^{1 *}$, QIANG ZHANG ${ }^{2 *}$, SHULONG ZHANG $^{3}$, HUICHUN LIU $^{1}$, \\ ZHONGRAN MAN $^{1}$ and YONG WANG ${ }^{1}$ \\ ${ }^{1}$ Department of Hepatobiliary Surgery; ${ }^{2}$ Clinical Laboratory, The First Affiliated Hospital, Bengbu Medical College, \\ Bengbu, Anhui 233004; ${ }^{3}$ Department of General Surgery, Zhongda Hospital, \\ Southeast University, Nanjing, Jiangsu 210009, P.R. China
}

Received July 24, 2016; Accepted October 10, 2018

DOI: $10.3892 / \mathrm{ol} .2018 .9740$

\begin{abstract}
Gallbladder cancer has a high recurrence and mortality rate, with limited treatment options. Therefore, elucidating the underlying molecular mechanisms of this disease would be beneficial to achieve an earlier diagnosis and potentially identify novel treatment targets. Claudin-1 is a tight junction protein associated with the development and prognosis of several types of cancer, and our preliminary studies have demonstrated that claudin-1 expression is elevated in gallbladder cancer tissues. Therefore, the aim of the present study was to investigate the effects of downregulating claudin-1 on the physiological processes of gallbladder cancer cells. The gallbladder cancer SGC996 cell line was transfected with claudin-1-RNA interference lentivirus (LV-CLDN1-RNAi) to downregulate claudin-1 expression, and the downstream effects on cell proliferation, the cell cycle, apoptosis and cell invasion were investigated. Following transfection with LV-CLDN1-RNAi, the results of an MTT assay revealed that downregulating claudin-1 did not affect the proliferation of the SGC996 cells. However, flow cytometry analysis demonstrated that the number of cells arrested in the $G_{1}$ phase increased significantly, whereas the amount of cells arrested in the $\mathrm{S}$ phase was significantly reduced. Annexin V-APC single-color staining demonstrated that downregulating claudin-1 expression increased the ratio of cell apoptosis, which was confirmed by the results of western blot analysis, in which levels of the pro-apoptotic B-cell lymphoma 2 (Bcl-2)-associated $\mathrm{X}$ protein and anti-apoptotic Bcl-2 protein
\end{abstract}

Correspondence to: Dr Hao Jin or Professor Huichun Liu, Department of Hepatobiliary Surgery, The First Affiliated Hospital, Bengbu Medical College, 287 Changhuai Road, Bengbu, Anhui 233004, P.R. China

E-mail: jinhaogandan@163.com

E-mail: liuhuichun2013@163.com

*Contributed equally

Key words: claudin-1, gallbladder cancer cells, cell proliferation, apoptosis, cell invasion were increased and decreased, respectively. Finally, a Transwell assay indicated that claudin-1 downregulation inhibited cell invasion. Overall, the results from the present study indicated that downregulating claudin-1 expression promoted the apoptosis of gallbladder cancer cells and inhibited cell invasion, indicating that claudin-1 may be involved in the recurrence and metastasis of gallbladder cancer. These insights provide theoretical and experimental foundations for considering claudin-1 as a novel target for the treatment of gallbladder cancer.

\section{Introduction}

Gallbladder cancer is a common malignant tumor of the digestive tract. The onset of gallbladder cancer is insidious, and the symptoms are difficult to detect at the early stages. Indeed, at the time that gallbladder tumors are clinically diagnosed, the cancer cells have mostly invaded the adjacent organs and metastasized via the lymph nodes. Compounding this challenge, gallbladder tumors are unresponsive to chemotherapy; therefore, early diagnosis and initiation of appropriate treatment are crucial for improving disease prognosis. In addition to the difficulty in the early detection and diagnosis of gallbladder cancer, recurrence and metastasis are important factors leading to the rapid progression of this disease, largely contributing to high mortality rates ( 0.45 cases per 100,000 individuals) (1). Several genes have been identified to be involved in the development of malignant tumors (2), indicating that further genetic studies may provide novel targets for the treatment of gallbladder cancer. Rapid cell proliferation and unregulated differentiation caused by abnormal tight junctions and damage to cell adhesion structures are important processes involved in tumorigenesis. Therefore, the loss or rearrangements of genes that encode tight junction proteins, including claudin or E-cadherin, are considered to be important triggers in the development of malignant tumors (3). Claudin-1 is an isoform of the tight junction protein family, and preliminary studies have demonstrated that claudin-1 expression is significantly increased in gallbladder cancer tissues, and is associated with cancer progression (4). However, these preliminary results were inconsistent with those from another previous study, which demonstrated that the expression of claudin-1 was significantly lower in gallbladder adenocarcinoma (5). Therefore, the present study was designed to evaluate 
the effects of downregulating claudin-1 on the physiological processes of the gallbladder cancer SGC996 cell line, including cell proliferation, apoptosis and invasion. The data from the present study are expected to provide potential novel strategies and approaches for the clinical development of targeted therapies for patients with gallbladder cancer.

\section{Materials and methods}

Cell lines and culture. The gallbladder cancer SGC996 and GBC-SD cell lines, and the cholangiocarcinoma QBC939 cell line were purchased from Shanghai GeneChem Co., Ltd. (Shanghai, China). SGC996 cells in the logarithmic growth phase were transfected with claudin-1-RNA interference lentivirus (LV-CLDN1-RNAi) (Shanghai GeneChem Co., Ltd.) using Lipofectamine 2000 (Invitrogen; Thermo Fisher Scientific, Inc., Waltham, MA, USA) to suppress claudin-1 expression. The negative-control cells were transfected with the virus CON077 synthesized by Shanghai GeneChem Co., Ltd. Restriction enzymes Agel and EcoRI (cat. nos. R3552L and R3101L, respectively) and T4 DNA ligase used for plasmid construction were purchased from New England BioLabs, Inc. (Ipswich, MA, USA). Following trypsin digestion (Sangon Biotech Co., Ltd., Shanghai, China), all cells were cultured in RPMI-1640 medium, supplemented with $10 \%$ fetal bovine serum (FBS; Invitrogen; Thermo Fisher Scientific, Inc.), $100 \mathrm{U} / \mathrm{ml}$ penicillin $\mathrm{G}$ and $100 \mu \mathrm{g} / \mathrm{ml}$ streptomycin. The cells were stored in an incubator at $5 \% \mathrm{CO}_{2}$ in a humidified atmosphere at $37^{\circ} \mathrm{C}$. The corresponding concentrations of the three cell lines were re-inoculated into culture plates to ensure the plated amounts were maintained at $15-30 \%$ during transfection.

Transfection of SGC996 cells with LV-CLDN1-RNAi. The cells were randomly divided into the experimental group transfected with LVpFU-GW-007PSC40161-1 (5'-GCAAAGTCTTTG ACTCCTTGC-3'; KD1 group), LVpFU-GW-007PSC40162-1 (5'-GCCACA AGACCTAGCCTAAT-3'; KD2 group), LVpFU-GW-007PSC40163-1 (5'-GCATCGTTATTAAGC CCTTAT-3'; KD3 group), the negative control (NC) transfected with CON077 (5'-TTCTCCGAACGTGTCACGT-3'), and the blank control (mock) group. Cells were transfected using Lipofectamine 2000 transfection reagent (Invitrogen; Thermo Fisher Scientific, Inc.), according to the manufacturer's protocols, and the concentration of lentivirus used in each well was $50 \mathrm{nM}$. An optimal amount of virus was used to initiate transfection, and culture medium was replaced at the optimal time, 8-12 h post-transfection. The cells were used for further experimentation $48 \mathrm{~h}$ after transfection. Analysis of transfected cells confirmed that cells, particularly NC and mock groups, were typically viable and apoptosis was rarely observed. In general, downstream applications were conducted if the transfection rate exceeded $70 \%$. The lentivirus was fluorescently labeled with green fluorescent protein (GFP). At $\sim 72 \mathrm{~h}$ post-transfection, fluorescence microscopy (IX71; Olympus Corporation, Tokyo, Japan) was performed to examine GFP reporter gene expression, and the rate of fluorescence indicated the rate of transfection. The lentivirus also harbored a resistant gene marker allowing for selection of successfully transfected cells. At $48-72$ h post-transfection, culture medium was replaced with a puromycin-containing culture medium (Clontech Laboratories, Inc., Mountainview, CA, USA), and the incubation was performed at $37^{\circ} \mathrm{C}$ overnight in a humidified atmosphere with $5 \% \mathrm{CO}_{2}$, to select for transfected cells, identified as highly proliferative cells. Positively identified transfected cells were used for downstream applications; otherwise, the transfection process was repeated. Each experiment represents a minimum of three independent repeats.

RNA extraction and reverse transcription-quantitative polymerase chain reaction $(R T-q P C R)$. Total RNA was extracted from cells using TRIzol reagent (Pufei Biotech Co, Ltd., Shanghai, China), according to the manufacturer's protocol. Total RNA was reverse transcribed to cDNA using a reverse transcription kit (Promega Corporation, Madison, WI, USA), according to the manufacturer's protocol. qPCR was performed using 10.0 $\mu \mathrm{l}$ SYBR premix Ex Taq (Takara Bio Inc., Otsu, Japan), $2 \mu \mathrm{l}$ forward primer $(2.5 \mu \mathrm{M}), 2 \mu \mathrm{l}$ reverse primer $(2.5 \mu \mathrm{M}), 2.0 \mu \mathrm{l} \mathrm{cDNA}$ and $4.0 \mu \mathrm{l}$ RNase-free $\mathrm{H}_{2} \mathrm{O}$. The primers were synthesized by RiboBio Co., Ltd., (Guangzhou, China), and the primer sequences used in the present study were as follows: GAPDH forward, 5'-TGACTTCAACAG CGACACCCA-3' and reverse, 5'-CACCCTGTTGCTGTA GCCAAA-3'; and claudin-1 (CLDN1) forward, 5'-AAA GTGAAGAAGGCCCGTATA-3' and reverse, 5'-TAATGT TGGTAGGGATCAAAGG-3'. The reaction conditions were as follows: Denaturation at $94^{\circ} \mathrm{C}$ for $10 \mathrm{~min}$, followed by 45 cycles of denaturation at $94^{\circ} \mathrm{C}$ for $15 \mathrm{sec}$, annealing at $60^{\circ} \mathrm{C}$ for $20 \mathrm{sec}$ and extension at $72^{\circ} \mathrm{C}$ for $15 \mathrm{sec}$. Duplicates were performed for all reactions using the Bio-Rad CFX96 real-time PCR machine (Bio-Rad Laboratories, Inc., Hercules, CA, USA). Melting curve analysis was performed and relative gene expression for each group was quantified by the $2^{-\Delta \Delta \mathrm{Cq}}$ method (6) as follows: $\Delta \mathrm{Cq}=\mathrm{Cq}$ value of the target gene- $\mathrm{Cq}$ value of the reference gene. At $\Delta \mathrm{Cq} \leq 12$, gene expression was considered to be high. At $12<\Delta \mathrm{Cq}<16$, gene expression was considered to be moderate. At $\Delta \mathrm{Cq} \geq 16$, gene expression was considered to be low. Relative gene expression was normalized to the endogenous housekeeping gene GAPDH. All experiments were performed in triplicate.

MTT assay for detecting cell proliferation. Cells in the logarithmic growth phase were digested with trypsin (Shanghai Chemical Reagent Co., Ltd., Shanghai, China) and re-suspended in complete medium (Ausbian; Shanghai Weizheng Xiangsheng Biotechnology Co., Ltd., Shanghai, China) for enumeration. The cells were seeded at a density of 2,000 cells/well and incubated at $37^{\circ} \mathrm{C}$ for $1,2,3,4$ or 5 days. The cell density was observed under a light microscope (XDS-100; Cai Kang Optical Instrument Co., Ltd, Shanghai, China). The number of plates was determined based on the experimental design (for example, if cells were to be detected for 5 days, then a total of 5 96-well plates were used). MTT (Genview; $20 \mu \mathrm{l}$ at $5 \mathrm{mg} / \mathrm{ml}$ ) was added to cells at $4 \mathrm{~h}$ prior to the termination of incubation. The culture medium was completely removed after $4 \mathrm{~h}$, ensuring the formazan particles that formed at the bottom of the plates remained undisturbed. The formazan particles were dissolved by adding $100 \mu \mathrm{l}$ DMSO. The mixtures were shaken for 2-5 min, and optical density values were measured at 490/570 nm using a microplate reader. Each group was replicated 3-5 times. 
Flow cytometry for cell cycle evaluation. Cells were treated by the aforementioned method, as described for the MTT assay. Cell suspensions were collected at a density of $\geq 1 \times 10^{6}$ in 5-ml centrifuge tubes, with triplicates for each group. The supernatant was discarded following centrifugation $(1,300 \mathrm{x} \mathrm{g}$ for $5 \mathrm{~min}$ at $4^{\circ} \mathrm{C}$ ), and cells were washed once with pre-chilled D-Hanks solution (pH, 7.2-7.4; Shanghai Jikai Gene Chemical Technology Inc.). Cells were fixed with $75 \%$ ethanol for $1 \mathrm{~h}$ prior to washing with D-Hanks solution. Cells were stained with 40X propidium iodide concentrate $(2 \mathrm{mg} / \mathrm{ml}$; Sigma-Aldrich; Merck KGaA, Darmstadt, Germany) at $37^{\circ} \mathrm{C}$ for $30 \mathrm{~min}$ and then stored in the dark at $4^{\circ} \mathrm{C}$. Cells were measured on a flow cytometer (Guava easyCyte HT; Merck KGaA) with the flow rate set at 300-800 cells/sec. The experiment was repeated three times, and the data were analyzed using the Modfit software (version 3.2; BD Biosciences, Franklin Lakes, NJ, USA).

Annexin V-APC single-color staining for detecting cell apoptosis. Cells were treated as aforementioned for the MTT assay, collected in 5-ml centrifuge tubes and re-suspended in complete medium for enumeration. Triplicate wells were set up for each group (to ensure sufficient cell numbers, the cell numbers were set at $\geq 5 \times 10^{5}$ for each treatment). Cells were centrifuged at $1,300 \mathrm{xg}$ for $5 \mathrm{~min}$ at $4^{\circ} \mathrm{C}$, and the supernatant was discarded. Cells were washed with D-Hanks solution, and with $1 \mathrm{X}$ binding buffer, and collected by re-centrifugation. A total of $200 \mu 11 \mathrm{X}$ binding buffer was used to re-suspend the cell pellet, and $10 \mu \mathrm{l}$ Annexin V-APC staining solution (eBioscience; Thermo Fisher Scientific, Inc.) was added. The mixture was added to $400-800 \mu 11 \mathrm{X}$ binding buffer based on the cell number. Cells were examined using a flow cytometer (Guava easyCyte HT; Merck KGaA) with the flow rate set at 300-800 cells/sec, the data were analyzed using the Modfit software, and the experiment was repeated three times.

Western blotting for apoptosis-associated proteins. Cells from different treatment groups were collected and treated with trypsin. Total protein was extracted using radioimmunoprecipitation assay buffer (Shanghai DingGuo Biotech Co., Ltd., Shanghai, China) and $1 \mu 1$ protease inhibitor per $1 \times 10^{6}$ cells, to lyse cells on ice for $30 \mathrm{~min}$. The mixture was centrifuged at $10,000 \times \mathrm{g}$ at $4^{\circ} \mathrm{C}$ for $10 \mathrm{~min}$, and protein quantification was performed using the Bradford method. The protein concentration of each sample was adjusted to $2 \mu \mathrm{g} / \mu \mathrm{l}$ and $20 \mu \mathrm{l}$ per sample was electrophoresed on $12 \%$ sodium dodecyl sulfate-polyacrylamide gel and the proteins were transferred onto a polyvinylidene difluoride membrane (EMD Millipore, Billerica, MA, USA). The membrane was blocked for $1 \mathrm{~h}$ in a closed shaker at room temperature. Membranes were probed with rabbit polyclonal anti-B-cell lymphoma-2 (Bcl-2; 1:800; cat. no. BS70205), rabbit polyclonal anti-Bcl-2-associated X (Bax; 1:500; cat. no. BS1030), and mouse anti-human $\beta$-actin (1:10,000; cat. no. BS6007M; all Bioworld Technology, Inc.). All primary antibodies were incubated overnight at $4^{\circ} \mathrm{C}$. Membranes were washed with TBS, and probed with secondary horseradish peroxidase-conjugated goat anti-rabbit (1:5,000; cat. no. KC-RB-035) or goat anti-mouse (1:5,000; KC-MM-035; both Kangcheng Biology Engineering Co., Ltd., Shanghai, China) IgG antibodies. The membrane was shaken at room temperature for $1 \mathrm{~h}$, washed with TBS, and chemiluminescence was obtained by exposing the membrane to X-rays. The ECL Western Blotting Substrate kit (Pierce; Thermo Fisher Scientific, Inc.) was used to visualize the bands (Image Lab version 4.0; Bio-Rad Laboratories, Inc.), and $\beta$-actin was used as an internal reference control.

Transwell assay for detecting cell invasion. Matrigel was melted at $4^{\circ} \mathrm{C}$ overnight and diluted to a ratio of 1:8 in RPMI-1640 culture medium. Diluted Matrigel $(50 \mu \mathrm{l})$ was added to the membrane of the upper Transwell chamber (pore size $8 \mu \mathrm{m}$; Corning Incorporated, Corning, NY, USA) of each well and dried at room temperature. Cells were collected, $48 \mathrm{~h}$ post-transfection and digested with trypsin, and cell density was adjusted to $1 \times 10^{5}$ cells $/ \mathrm{ml}$. A total of $200 \mu \mathrm{l}$ of the cell suspension was inoculated in the upper chamber, and $600 \mu \mathrm{l}$ of RPMI-1640 culture medium containing $10 \%$ FBS was added to the lower chamber as the chemoattractant. Following incubation at $37^{\circ} \mathrm{C}$ and $5 \% \mathrm{CO}_{2}$ in air for $48 \mathrm{~h}$, the Transwell chamber was removed and the culture medium was eliminated. A cotton swab was used to wipe off the Matrigel from the upper Transwell chamber and the residual cells. The invading cells were then fixed using $95 \%$ ethanol for $5 \mathrm{~min}$, and stained with $0.1 \%$ crystal violet for $15 \mathrm{~min}$, both at room temperature. Cells that migrated through the inserts were counted on 10 different randomly chosen fields of view per inset under a light microscope (Nikon Corporation), at magnification, x100. The experiment was repeated three times.

Statistical analysis. Statistical analysis of all data was performed using SPSS 21.0 (IBM Corp., Armonk, NY, USA). Data are expressed as the mean \pm standard deviation. Comparisons of multiple groups were performed using a one-way analysis of variance with a least significant difference post hoc test. $\mathrm{P}<0.05$ was considered to indicate a statistically significant difference.

\section{Results}

Effect of LV-CLDN1-RNAi transfection on claudin-1 expression. The qPCR results demonstrated that claudin-1 mRNA was highly expressed in QBC939 and SGC996 cells, but only moderately expressed in GBC-SD cells (Table I; Fig. 1). The QBC939 cell line is from human cholangiocarcinoma cells; therefore, the gallbladder SGC996 cell line, which displayed high claudin-1 mRNA expression, was selected for subsequent functional studies. Furthermore, claudin-1 mRNA expression levels in SGC996 cells transfected with LV-CLDN1-RNAi in the KD2 group were significantly lower $(\mathrm{P}=0.0002)$ than those in control cells. The overall knockout efficiency was $84.4 \%$ (Fig. 2).

\section{Effect of claudin-1 downregulation on cell proliferation and the} cell cycle. Analysis of cell proliferation by MTT assay demonstrated that in response to transfection with LV-CLDN1-RNAi after 1,2, 3 and 4 days, the proliferation rate of SGC996 cells was not significantly different compared with that of the control group $(\mathrm{P}>0.05$; Fig. $3 \mathrm{~A})$. Although the Kruskal-Wallis test yielded a non-significant result $(\mathrm{P}>0.05)$, the difference in the growth rate was $<20 \%$, identified as negative, indicating no major effect on cell proliferation. By contrast, flow cytometry analysis demonstrated that the proportion of SGC996 cells in the $G_{1}$ phase in the KD group was significantly increased compared with that in the NC group $(\mathrm{P}=0.000013)$, while the 
Table I. $\Delta$ Cq of SGC996, GBC-SD and QBC939 cells showing high expression of claudin-1 mRNA in QBC939 and SGC996 cells, but moderate expression in GBC-SD cells. ${ }^{a}$

\begin{tabular}{lccr}
\hline Cell line & GAPDH & Claudin-1 & $\Delta$ Cq \\
\hline SGC996 & 14.46 & 20.99 & 6.53 \\
& 14.49 & 20.97 & 6.48 \\
GBC-SD & 14.66 & 20.94 & 6.28 \\
& 13.96 & 28.21 & 14.25 \\
& 14.07 & 28.51 & 14.44 \\
QBC939 & 14.08 & 28.36 & 14.28 \\
& 16.49 & 19.35 & 2.86 \\
& 16.52 & 19.48 & 2.96 \\
& 16.44 & 19.49 & 3.05 \\
\hline
\end{tabular}

${ }^{\text {aSGC}} 996$ cells, in which the expression of claudin-1 mRNA was high, was chosen for subsequent functional studies.

proportion of cells in the $\mathrm{S}$ phase was significantly decreased $(\mathrm{P}=0.0004)$. There were no significant differences between groups for cells in the $\mathrm{G}_{2} / \mathrm{M}$ phase ( $\mathrm{P}>0.05$ ) (Fig. 3B).

Effectofclaudin-1 downregulation onapoptosis. Annexin V-APC single-color staining revealed that downregulation of claudin-1 led to significantly increased apoptosis in the KD group compared with that in the NC group ( $\mathrm{P}<0.05$; Fig. 4A). Similarly, western blot analysis revealed that the downregulation of claudin-1 led to increased apoptotic protein Bax expression and decreased anti-apoptotic protein Bcl-2 expression (Fig. 4B).

Effect of claudin-1 downregulation on cell invasion. A Transwell invasion assay was conducted to assess cells that traverse across a porous Matrigel membrane in order to reach the lower chamber. As Matrigel mimics the in vivo extracellular matrix, the assay may be applied to determine the invasion capacity of gallbladder cells. The results demonstrated that the invasion capacity of the KD group was significantly inhibited compared with that of the NC group (Fig. 5).

\section{Discussion}

Claudin proteins contribute important functions in tight junctions and the maintenance of epithelial cell polarity. To date, 24 subtypes of claudin have been identified, of which CLDNI is located on the human chromosome 3q28-q29 locus. CLDN1 encodes a subtype of tight-junction protein, which consists of 211 amino acids and is mainly involved in the regulation of cell-cell adhesion (7). Previous studies have reported that claudin-1 expression may be upregulated or ectopically expressed in tumors, implicating a role in specific physiological processes during tumor development $(7,8)$. The functions of claudin-1 in tumor development have been previously reported in multiple studies (9-11). Suren et al (12) observed that claudin-1 expression was not altered in nose cancer tissues compared with non-cancer epithelial tissues, and no association with tumor prognosis was determined. However, Claudin-1 was demonstrated to be highly upregulated in oral squamous cell carcinoma, which was

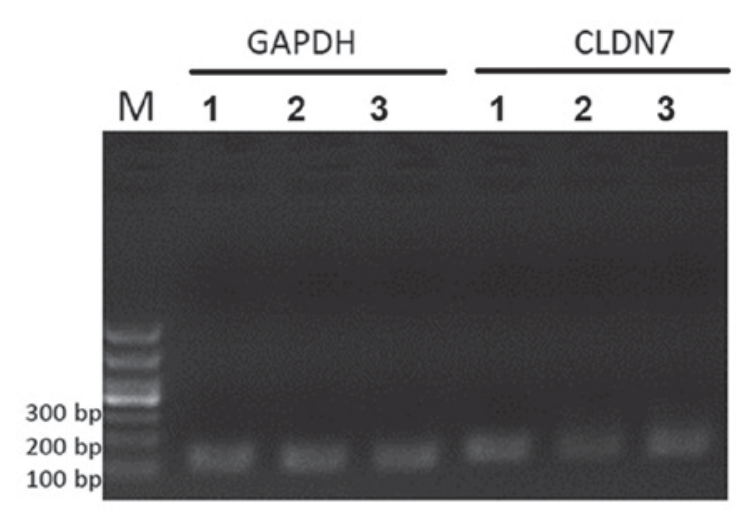

Figure 1. Expression of claudin-1 mRNA in SGC996, GBC-SD and QBC939 cells. Lane 1, QBC939 cells; lane 2, SGC996 cells; lane 3, GBC-SD cells; M, DNA ladder; GAPDH, glyceraldehyde 3-phosphate dehydrogenase.

associated with histological grades, vascular invasion, lymph node metastasis and clinical stages, which further indicated a poor prognosis (13). Holczbauer et al (14) performed immunohistochemistry and western blotting to detect claudin-1 expression in liver cancer tissues, liver cirrhosis tissues and normal liver tissues. Claudin-1 expression was significantly increased in liver cancer and hepatitis B-associated liver cirrhosis tissues, but did not differ between normal tissues and hepatitis $\mathrm{C}$-associated liver cirrhosis tissues. These results suggested that claudin-1 is able to promote the invasion of hepatitis B virus and epithelial-mesenchymal transition. Bouchagier et al (15) also observed that increased claudin-1 expression in liver cancer tissues was a positive prognostic indicator, suggesting that it may be associated with a positive treatment effect and high survival rate.

Claudin-1 has also been demonstrated to be associated with colon cancer. Matsuoka et al (16) reported that upregulated claudin-1 expression was linked with tumor invasion and development in colon cancer. Furthermore, claudin-1 expression was significantly increased in colon cancer that had metastasized. The study suggested a possible mechanism by which claudin-1 expression is initially reduced during the early stage of tumor formation in the absence of metastasis, but subsequently increases during tumor development, alongside a decrease in E-cadherin expression, triggering the accumulation of $\beta$-catenin in the cell. Upregulation of the $\beta$-catenin/TCF signaling pathway may further promote claudin- 1 expression, causing damage to the structure and function of tight junction proteins. This results in a decrease in tissue adhesion or causes damage to adhesion molecules, allowing cancer cells to easily detach from their primary tumor site. The detached cancer cells are able to adhere to the interstitial or intercellular membranes of normal cells, blood and lymphatic vessels. The enhanced migration capacity of the cancer cells further promotes their infiltration, ultimately resulting in metastasis. Süren et al (17) demonstrated that reduced claudin-1 expression was highly correlated with the degree of tumor invasion, metastasis, vascular invasion and perineural invasion in colorectal carcinoma. Furthermore, Guo et al (18) revealed that claudin-1 expression in cholangiocarcinoma was associated with tumor invasion and metastasis.

During our preliminary study, prior to the present study, it was revealed that claudin-1 expression was significantly upregulated in gallbladder cancer tissues (4). However, this observation differed from that of another study (5), and the 


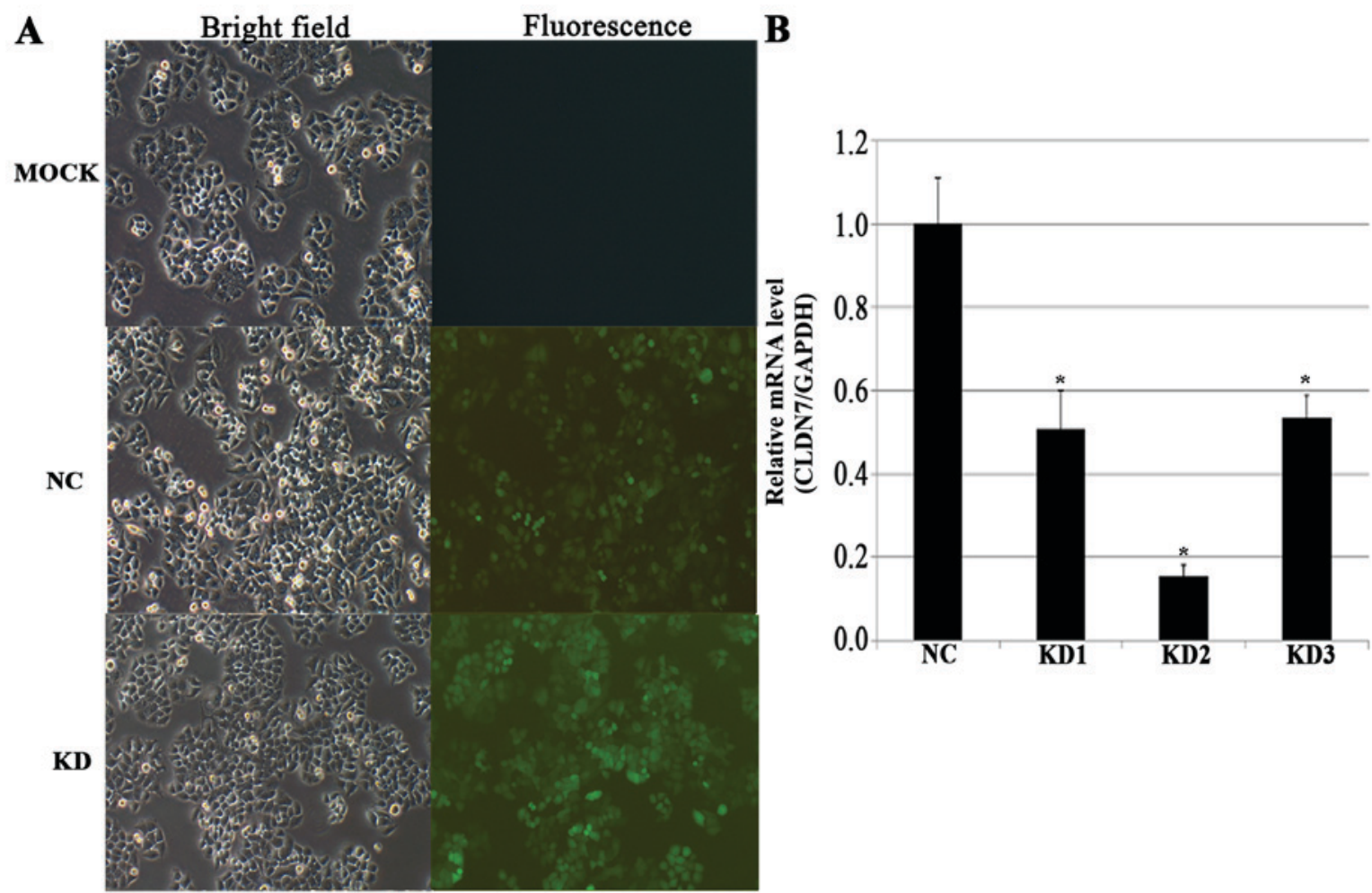

Figure 2. Fluorescence images and claudin-1 mRNA expression in SGC996 cells transfected with LV-CLDN1-RNAi. (A) Fluorescence images of cells pre- and post- transfection (magnification, x100). (B) Expression of claudin-1 mRNA in SGC996 cells transfected with LV-CLDN1-RNAi. "P<0.05 vs. NC. NC, negative control; CLDN7, claudin-7; GAPDH, glyceraldehyde 3-phosphate dehydrogenase; KD, knockdown; LV-CLDN7-RNAi, claudin-1-RNA interference lentivirus.


Figure 3. Analysis of SGC996 cell proliferation and cell cycle. (A) Proliferation curve of SGC996 cells transfected with a blank control vector (mock), NC vector or LV-CLDN1-RNAi to downregulate claudin-1 expression (KD). (B) Analysis of cell cycle changes in SGC996 cells transfected with blank control vector (mock), negative control (NC) vector or LV-CLDN1-RNAi to downregulate claudin-1 expression (KD). Flow cytometry analysis demonstrated that the proportion of SGC996 cells in the $\mathrm{G}_{1}$ phase in the KD group was significantly increased, while the proportion of cells in the S phase was significantly decreased compared with that of the NC group. "P<0.05 vs. NC. LV-CLDN1-RNAi, claudin-1-RNA interference lentivirus; NC, negative control; KD, knockdown; OD, optical density.

exact mechanisms involved remain unclear. Therefore, the present study was designed to specifically examine the effects of claudin-1 on the behavior and proliferation of cancer cells by downregulating its expression by RNAi.

The results from the MTT assay demonstrated that downregulated claudin-1 did not affect cell proliferation; however, flow cytometry results revealed that a large proportion of cells were arrested in the $\mathrm{G}_{0} / \mathrm{G}_{1}$ phase. Furthermore, downregulating claudin-1 in SGC996 cells inhibited the transition of the $\mathrm{G}_{1} / \mathrm{S}$ phase in gallbladder cancer cells. Downregulation of claudin-1 promoted the apoptosis and inhibited the invasion ability of gallbladder cancer cells in vitro.

The results from the present study in gallbladder cells are consistent with those reported by Shiozaki et al (19) in 


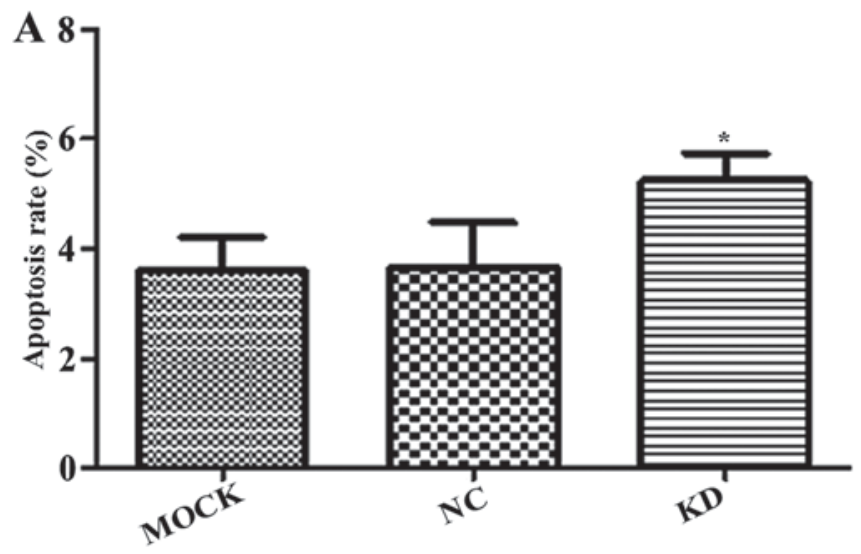

B

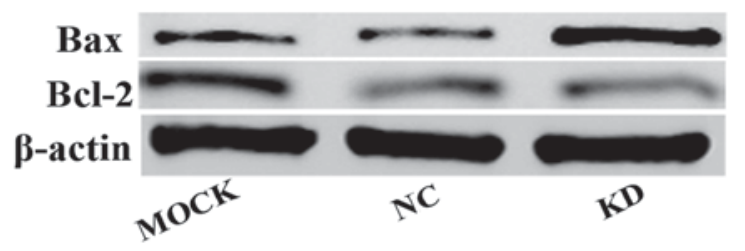

Figure 4. Analysis of the apoptosis rate and apoptosis-related protein expression in cells transfected with a blank control vector (mock), NC vector or LV-CLDN1-RNAi. (A) Changes in apoptosis in cells transfected with a blank control vector (mock), negative control (NC) vector or LV-CLDN1-RNAi to downregulate claudin-1 expression (KD). (B) Western blotting of apoptosis-associated proteins in cells transfected with a blank control vector (mock), negative control (NC) vector or LV-CLDN1-RNAi to downregulate claudin-1 expression (KD). " $\mathrm{P}<0.05$ vs. NC. LV-CLDN1-RNAi, claudin-7-RNA interference lentivirus; $\mathrm{NC}$, negative control; KD, knockdown.
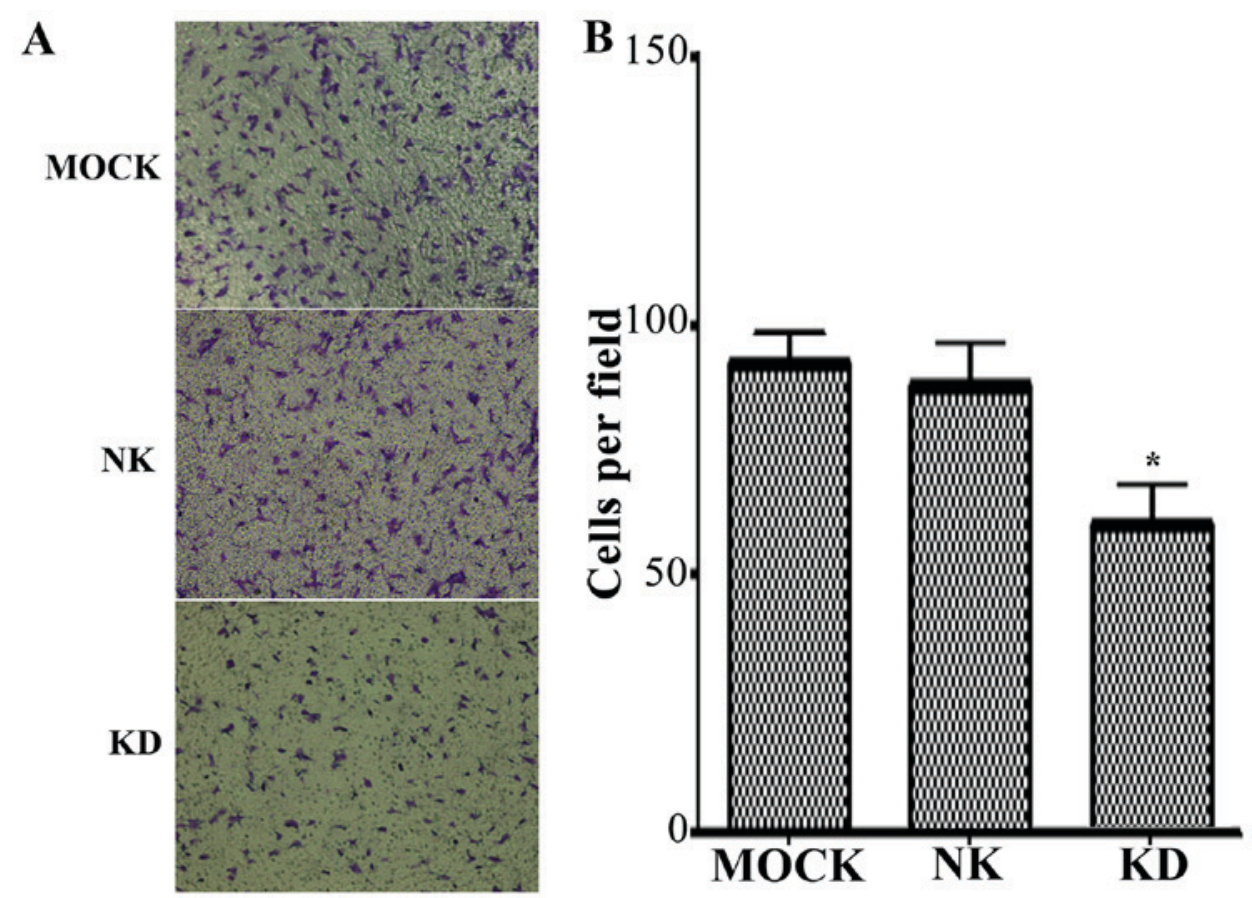

Figure 5. Invasive ability of cells transfected with a blank control vector (Mock), NC vector or LV-CLDN1-RNAi to downregulate claudin-1 expression (KD), A Transwell assay was conducted in which the cells had to traverse across the porous Matrigel membrane in order to reach the lower chamber. As Matrigel mimics the in vivo extracellular matrix, this assay could be used to determine the invasion capability of the gallbladder cells. (A) Representative images showing the migration of cells transfected with a blank control vector (Mock), NC vector or LV-CLDN1-RNAi to downregulate claudin-1 expression (KD) (magnification, $\mathrm{x} 100$ ). (B) The number of migrated cells was quantified. The results showed that the invasion ability of the KD group was inhibited. ${ }^{*} \mathrm{P}<0.05$ vs. NC.LV-CLDN1-RNAi, claudin-1-RNA interference lentivirus; NC, negative control; KD, knockdown.

gastric cancer, in which downregulated claudin-1 inhibited the proliferation, invasion and metastasis of gastric cancer cells, yet promoted apoptosis. A possible mechanism was proposed, such that downregulation of CLDN1 may induce tumor necrosis factor- $\alpha$ (TNF- $\alpha$ ) expression, which in turn altered the expression of 245 genes, including those associated with cell motility, including matrix metallopeptidase 7 (MMP7), TNF (ligand) superfamily member 10, transforming growth factor $\beta$ receptor- 1 and $\mathrm{C}-\mathrm{C}$ motif chemokine 2 (CCL2). Shiozaki et al (20) also suggested that downregulated claudin-1 promoted apoptosis in human lung cancer cells through the induction of TNF- $\alpha$ expression. Typically, as a tight junction protein involved in cell adhesion, downregulation of claudin-1 would be likely to promote cell migration. However, the results from the present study are in contrast with this expectation, suggesting a mechanism of induced expression of cytoplasmic TNF- $\alpha$, which further activates a series of signaling pathways, ultimately leading to apoptosis (20). Pope et al (21) demonstrated that overexpression of claudin-1 accelerated tumor growth and development, and was indicative of a poor prognosis. A possible mechanism for this effect is via the activation of the Wnt signaling pathway. 
The results from the present study were therefore consistent with those from previous studies, although the lack of significant changes in cell proliferation in response to downregulating claudin-1 expression remains to be addressed, which could be due to experimental error. In addition, further studies on a series of the potential molecular mechanisms in a state of downregulated claudin-1 expression in gallbladder cancer are required.

In summary, the present results demonstrated that claudin-1 serves an important role in the proliferation, apoptosis and invasion of gallbladder cancer cells, as well as in the carcinogenesis and development of gallbladder cancer. Identification of the signaling pathways triggered by claudin-1, as well as the molecular mechanisms involved, is required in follow-up studies. Understanding a clear mechanism for these effects will likely contribute toward developing claudin-1 as a novel potential target for the treatment of gallbladder cancer.

\section{Acknowledgements}

Not applicable.

\section{Funding}

The present study was supported by funding from the Anhui Provincial Education Department (grant no. KJ2015B115BY).

\section{Availability of data and materials}

The datasets used and/or analyzed during the current study are available from the corresponding author on reasonable request.

\section{Authors' contributions}

HJ, QZ and SZ wrote the manuscript and conducted the experiments; HL designed the present study and revised the manuscript; ZM and YW performed data analysis.

\section{Ethics approval and consent to participate}

Not applicable.

\section{Patient consent for publication}

Not applicable.

\section{Competing interests}

The authors declare that they have no competing interests.

\section{References}

1. Wang JH and Jiang XQ: The epidemiological studies of primary gallbladder carcinoma. Chin Arch Gen Surg (Electronic Edition) 4: 271-273, 2010.

2. Kunert-Keil C,Steinmüller F, Jeschke U, Gredes T and Gedrange T: Immunolocation of glycodelin in human adenocarcinoma of the lung, sqamous cell carcinoma of the lung and lung metastases of colonic adenocarcinoma. Acta Histochem 113: 798-802, 2011.

3. Sánchez-Tilló E, de Barrios O, Siles L, Cuatrecasas M, Castells A and Postigo A: $\beta$-catenin/TCF4 complex induces the epithelial-to-mesenchymal transition(EMT)-activator ZEB to regulate tumor invasiveness. Proc Natl Acad Sci USA 108: 19204-19209, 2011.
4. Jin H, Liu HC, Zhou SB and Yu DH: Expression of claudin-1 in gallbladder carcinoma and its significance. J Bengbu Med Coll: 22-24, 2012.

5. Xiong L, Wen Y, Miao X and Yang Z: Expressions of cell junction regulatory proteins and their association with clinicopathologic parameters in benign and malignant gallbladder lesions. Am J Med Sci 342: 388-394, 2011.

6. Svec D, Tichopad A, Novosadova V, Pfaffl MW and Kubista M: How good is a PCR efficiency estimate: Recommendations for precise and robust qPCR efficiency assessments. Biomol Detect Quantif 3: 9-16, 2015.

7. Hewitt KJ, Agarwal R, Morin PJ: The claudin gene family: Expression in normal and neoplastic tissues. BMC Cancer 6: 186, 2006.

8. Kominsky SL: Claudins: Emerging targets for cancer therapy. Expert Rev Mol Med 8: 1-11, 2006.

9. Gruber R, Börnchen C, Rose K, Daubmann A, Volksdorf T, Wladykowski E, Vidal-Y-Sy S, Peters EM, Danso M, Bouwstra JA, et al: Diverse regulation of claudin-1 and claudin-4 in atopic dermatitis. Am J Pathol 185: 2777-2789, 2015.

10. Hahn-Strömberg V, Askari S, Befekadu R, Matthiessen P, Karlsson S and Nilsson TK: Polymorphisms in the CLDN1 and CLDN7 genes are related to differentiation and tumor stage in colon carcinoma. APMIS 122: 636-642, 2014.

11. Morgan C, Jenkins SA, Kynaston HG and Doak SH: The role of adhesion molecules as biomarkers for the aggressive prostate cancer phenotype. PLoS One 8: e81666, 2013.

12. Suren D, Yildirim M, Kaya V, Elal R, Selcuk OT, Osma U, Yildiz M, Gunduz S and Sezer C: Expression patterns of claudins 1,4 , and 7 and their prognostic significance in nasopharyngeal carcinoma. J BUON 20: 212-217, 2015.

13. Sappayatosok K and Phattarataratip E: Overexpression of claudin-1 is associated with advanced clinical stage and invasive pathologic characteristics of oral squamous cell carcinoma. Head Neck Pathol 9: 173-180, 2015.

14. Holczbauer Á, Gyöngyösi B, Lotz G, Törzsök P, Kaposi-Novák P, Szijártó A, Tátrai P, Kupcsulik P, Schaff Z and Kiss A: Increased expression of claudin-1 and claudin-7 in liver cirrhosis and hepatocellular carcinoma. Pathol Oncol Res 20: 493-502, 2014.

15. Bouchagier KA, Assimakopoulos SF, Karavias DD, Maroulis I, Tzelepi V, Kalofonos H, Karavias DD, Kardamakis D, Scopa CD and Tsamandas AC: Expression of claudins-1, -4, -5, -7 and occludin in hepatocellular carcinoma and their relation with classic clinicopathological features and patients' survival. In Vivo 28: 315-326, 2014.

16. Matsuoka T, Mitomi H, Fukui N, Kanazawa H, Saito T, Hayashi T and Yao T: Cluster analysis of claudin-1 and 4, E-eadhefin, and $\beta$-catenin expression in colorectal cancers. J Surg Oncol 103: 674-686, 2011.

17. Süren D, Yıldırım M, Kaya V, Alikanoğlu AS, Bülbüller N, Ylldlz M and Sezer C: Loss of tight junction proteins (Claudin 1,4 , and 7) correlates with aggressive behavior in colorectal carcinoma. Med Sci Monit 20: 1255-1262, 2014.

18. Guo XJ, Wang M, Jiang JX, Shi CJ, Hu J and Huang HJ: Expression of zonula occludens- 1 and Claudin-1 and it's significance in cholangiocarcinoma. Chin J Exp Surg 31: 1739-1741, 2014 (In Chinese).

19. Shiozaki A, Shimizu H, Ichikawa D, Konishi H, Komatsu S, Kubota T, Fujiwara H, Okamoto K, Iitaka D, Nakashima S, et al: Claudin 1 mediates tumor necrosis factor alpha-induced cell migration in human gastric cancer cells. World J Gastroenterol 20: 17863-17876, 2014.

20. Shiozaki A, Bai XH, Shen-Tu G, Moodley S, Takeshita H, Fung SY, Wang Y, Keshavjee S and Liu M: Claudin 1 mediates TNF- $\alpha$-induced gene expression and cell migration in human lung carcinoma cells. PLoS One 7: e38049, 2012.

21. Pope JL, Ahmad R, Bhat AA, Washington MK, Singh AB and Dhawan P: Claudin-1 overexpression in intestinal epithelial cells enhances susceptibility to adenamatous polyposis coli-mediated colon tumorigenesis. Mol Cancer 13: 167, 2014.

This work is licensed under a Creative Commons Attribution-NonCommercial-NoDerivatives 4.0 International (CC BY-NC-ND 4.0) License. 